\title{
Magnetic particle motion in a Poiseuille flow
}

\author{
J. W. Haverkort, ${ }^{*}$ S. Kenjereš, and C. R. Kleijn \\ Department of Multi-Scale Physics, Delft University of Technology, Prins Bernhardlaan 6, 2628 BW Delft, The Netherlands \\ and J. M. Burgers Centre for Fluid Mechanics, 2628 CD Delft, The Netherlands
}

(Received 8 March 2009; revised manuscript received 25 May 2009; published 6 July 2009)

\begin{abstract}
The manipulation of magnetic particles in a continuous flow with magnetic fields is central to several biomedical applications, including magnetic cell separation and magnetic drug targeting. A simplified twodimensional (2D) equation describing the motion of particles in a planar Poiseuille flow is considered for various magnetic field configurations. Exact analytical solutions are derived for the particle motion under the influence of a constant magnetization force and a force decaying as a power of the source distance, e.g., due to a current carrying wire or a magnetized cylinder. For a source distance much larger than the transversal size of the flow, a general solution is derived and applied to the important case of a magnetic dipole. This solution is used to investigate the dependence of the particle capture efficiency on the dipole orientation. A correction factor to convert the obtained 2D results to a three-dimensional cylindrical geometry is derived and validated against computational simulations. Simulations are also used to investigate parameter ranges beyond the region of applicability of the analytical results and to investigate more complex magnetic field configurations.
\end{abstract}

DOI: 10.1103/PhysRevE.80.016302 PACS number(s): 47.63.mh, 87.85.Tu, 45.50.Dd, 87.10.Ca

\section{INTRODUCTION}

The ability to manipulate magnetic particles in fluid flows by means of inhomogeneous magnetic fields is used in a wide range of industrial and biomedical applications. An example is high gradient magnetic separation (HGMS) which includes the processing of minerals and the treatment of wastewater [1]. Magnetic particles have a variety of biomedical applications, e.g., for hyperthermia treatment, for cell separation, as contrast agents for magnetic resonance imaging (MRI), or in magnetic drug targeting (MDT). In the latter application, the localized delivery of chemotherapeutic agents $[2,3]$ is enhanced by attaching them to magnetic particles which can be attracted to a tumor by an external magnetic field. Application to cardiovascular disease is currently under investigation. Knowledge of the particle trajectories in an applied magnetic field can help estimate the fraction of particles captured for a specific piece of artery, thereby allowing an a priori estimate of the effectiveness of a specific treatment. Significant theoretical [4-6], experimental [7-10], and computational [11-14] progress in this direction has been made over the past years. With immunomagnetic separation, DNA, cells, organelles, proteins, etc. can be isolated from a fluid for the purpose of, e.g., medical diagnosis. In recent years an increased interest has arisen in applying this technique to a continuously flowing fluid [15]. Continuous flow magnetic separation is often investigated in microfluidic devices, in which it can easily be integrated in-line with other processing steps of a micro total analysis system ( $\mu$ TAS), and components can be laterally separated depending on the component's sizes and magnetic content.

In all mentioned applications, detailed knowledge of the particle trajectories is needed for the design and application of the devices. Due to the geometric complexity of both the flow and the magnetic field, predictions of these trajectories

\footnotetext{
*j.w.haverkort@cwi.nl
}

are in general obtained from numerical simulations. Analytical expressions for the particle trajectories can be obtained for relatively simple geometries and under some simplifying assumptions only. These simplifications are not generally valid in, e.g., microfluidic devices for bioseparation, which typically have multiple embedded magnetic source elements integrated into the device close to the fluid channel $[16,17]$.

Nevertheless, there is a clear use and need for such analytical solutions. First of all, they can be used to validate numerical simulation codes. Second, they provide insight into the general scaling characteristics of particle movement in magnetic fields that also hold for more complex geometries. This is useful in explaining for instance the scaling behavior found in $[9,10]$. Finally, although derived for simple geometries, such analytical solutions may serve as good approximations for the particle movement in more complicated devices. As an example, knowledge of the behavior of magnetic particles in laminar flow under the influence of a circular current loop may help in the description of the effectiveness of magnetic traps such as used in [18] to focus single particles.

In this work we derive analytical expressions for the trajectories of magnetic particles in Poiseuille flows for a range of simple but generic magnetic fields. We ignore particleparticle (e.g., dipole-dipole) interactions, as well as the effect of the particles on the flow. These are valid approximations for systems with small particle volume fractions, as in most of the aforementioned applications.

Exact analytical solutions are derived for the particle motion in a planar Poiseuille flow in a constant or radial magnetization force field. These solutions are for instance relevant for fluid devices in which magnetic fields are generated by permanent magnetic elements or electromagnets outside the fluid. The presented result for a line force parallel to the flow is relevant for applications such as in [9], where two magnetic wires are used for the ex-vivo filtering of human blood from magnetic drug-loaded or toxin-loaded spheres. It might also be of use for applications with a conducting wire mesh such as in [19], as an approximate result for when the 
distance between the wires is large. The presented solution for a magnetic monopole is relevant for applications in which spiked magnetic elements are used, such as in [20].

We also present an approximate solution for a planar Poiseuille flow and a generic magnetic field under the assumption that the source distance is much larger than the transversal size of the flow. This is a valid assumption for applications such as magnetic drug targeting, in which the magnetic source is usually placed outside the body at a distance of several centimeters from the treated artery. The obtained approximate solution is applied to the fundamental case of magnetic particle motion in a planar Poiseuille flow in the field of a magnetic dipole, for which the fraction of captured particles is investigated as a function of the dipole orientation.

Finally, again under the assumption that the source distance is much larger than the transversal size of the flow, we derive a correction to transform the obtained results for the planar Poiseuille flow into expressions for the Poiseuille flow in a straight cylindrical tube. Three-dimensional computational simulations are used to validate and extend the analytical results.

\section{MATHEMATICAL FORMULATION}

\section{A. Magnetization force}

Particles above a critical size (tens of nanometers for the most frequently used materials iron, magnetite and hematite) consist of several magnetic domains. The magnetization behavior of these multidomain particles is fairly well described by a model in which the magnetization $\mathbf{M}$ below saturation is linear in the auxiliary magnetic field $\mathbf{H}[21]$

$$
\mathbf{M}=\left\{\begin{array}{cl}
\chi \mathbf{H} & H<M_{s a t} /|\chi| \\
(\chi /|\chi|) M_{s a t} \hat{\mathbf{H}} & H \geq M_{\text {sat }} /|\chi|,
\end{array}\right.
$$

where $\chi$ is the magnetic susceptibility, $M_{\text {sat }}$ is the saturation magnetization, and a hat is used to denote a unit vector, i.e., $\hat{\mathbf{H}}=\mathbf{H} / H$. The magnitude of a vectorial quantity will be denoted by the same symbol without applied boldface, e.g., $H=|\mathbf{H}|$. In this work we adopt the linear magnetization model of Eq. (1) and assume that the magnetization instantaneously aligns with the applied magnetic field. The magnetization force, which is also known as the magnetophoretic force or the Kelvin force, exerted on a magnetized material is per unit volume given by $\mathbf{f}_{m}=\mu_{0} \mathbf{M} \cdot \boldsymbol{\nabla H}$. Using the linear magnetization model of Eq. (1) we obtain

$$
\mathbf{f}_{m}= \begin{cases}\mu_{0} \chi \nabla H^{2} / 2 & H<M_{\text {sat }} /|\chi| \\ (\chi /|\chi|) \mu_{0} M_{\text {sat }} \nabla H & H \geq M_{\text {sat }} /|\chi|,\end{cases}
$$

where use has been made of the relation $\boldsymbol{\nabla} H^{2} / 2=\mathbf{H} \times(\boldsymbol{\nabla}$ $\times \mathbf{H})+(\mathbf{H} \cdot \boldsymbol{\nabla}) \mathbf{H}$ and the assumption that $\mathbf{H}$ is curl free.

\section{B. Particle equations of motion}

The motion of spherical particles of diameter $D$ in a steady laminar Poiseuille flow will be considered. Assuming low particle concentrations, the interaction between particles and the effect of the particles on the fluid can be neglected.

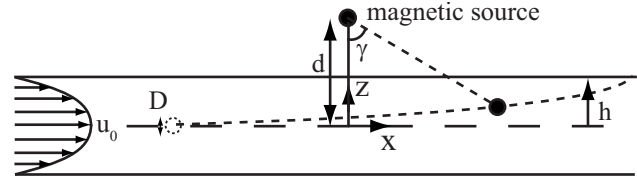

FIG. 1. A schematic overview of the considered geometry, coordinates, and geometrical variables.

The considered two-dimensional geometry is shown in Fig. 1. The maximum flow velocity is $u_{0}$, the channel height is $h$, and the distance from a magnetic source to the flow centerline is $d$. An overbar on a parameter with dimension of length will be used to denote nondimensionalization with $h$, i.e., $\bar{d}=d / h, \bar{D}=D / h$, etc.

When necessary to avoid confusion, the subscripts $f$ and $p$ will be used to denote the fluid and the particles, respectively. We will assume that the particle Reynolds number $\operatorname{Re}_{p}=\left|\mathbf{u}_{p}-\mathbf{u}_{f}\right| D / \nu \ll 1$, where $\nu=\mu / \rho_{f}$ is the fluid kinematic viscosity and $\mathbf{u}_{p}$ and $\mathbf{u}_{f}$ as the particle and the fluid velocities, respectively. We will also assume the particles are much smaller than the channel height, i.e., $\bar{D} \ll 1$. Because the ratio $\left(\rho_{p} / 18 \rho_{f}\right) \operatorname{Re}_{p} \bar{D}$ between the particle relaxation time $\rho_{p} D^{2} / 18 \mu$ and the time scale $h /\left|\mathbf{u}_{p}-\mathbf{u}_{f}\right|$ of the particle motion becomes negligible for $\operatorname{Re}_{p} \ll 1$ and $\bar{D} \ll 1$, we can describe the particle motion in terms of an equilibrium of forces:

$$
0=\mathbf{F}_{D}+\mathbf{F}_{m}+\mathbf{F}_{L}+\mathbf{F}_{g} .
$$

(i) $\mathbf{F}_{D}$ : for $\operatorname{Re}_{p} \ll 1$, the drag force $\mathbf{F}_{D}$ can be modeled using Stokes' expression $\mathbf{F}_{D}=3 \pi \mu D\left(\mathbf{u}_{f}-\mathbf{u}_{p}\right)$.

(ii) $\mathbf{F}_{m}$ : when the magnetic field is approximately constant over the volume of a particle and the surrounding medium has a negligible magnetic susceptibility, the magnetic force $\mathbf{F}_{m}$ on a magnetic particle can be evaluated by multiplying the magnetization force density from Eq. (2) by the particle volume $\pi D^{3} / 6$ [4].

(iii) $\mathbf{F}_{L}$ : the lift forces $\mathbf{F}_{L}$ on a spherical particle are associated with velocity gradients in the fluid, in particular the Faxen force and the Saffman lift force. The magnitude of the Faxen force at the centerline of a Poiseuille flow is given by $1.05235 \bar{D} F_{D}$ for $\operatorname{Re}_{p} \ll 1$ and $\bar{D} \ll 1$ [22]. The magnitude of the Saffman lift force at a distance $s=\bar{s} h$ from the centerline of a Poiseuille flow can be written as $(K / 3 \sqrt{2} \pi) \sqrt{\bar{s} \operatorname{Re}_{p} \bar{D}} F_{D}$, with $K=1.61$ for $\operatorname{Re}_{p} \ll 1$ and the channel Reynolds number $\operatorname{Re} \gg 1 / \bar{s}$ [23]. It follows that on an integral scale, thereby excluding the region close to the wall, lift effects can be neglected for $\operatorname{Re}_{p} \ll 1$ and $\bar{D} \ll 1$.

(iv) $\mathbf{F}_{g}$ : the ratio between the gravitational force density $\rho_{p} g$ and the magnetization force density $\left|\mu_{0} M \nabla H\right|$ is generally very small in applications with magnetic particles, such that the gravitational force $\mathbf{F}_{g}$ can be neglected.

The conditions $\operatorname{Re}_{p} \ll 1$ and $\bar{D} \ll 1$, for which the Stokes drag force can be used and inertial and lift effects can be neglected, allow for the description of most biomedical applications. In this case the equations of motion simplify to a 
balance between the drag force and the magnetization force $\mathbf{F}_{m}$

$$
0=3 \pi \mu D\left(\mathbf{u}_{f}-\mathbf{u}_{p}\right)+\mathbf{F}_{m} .
$$

It follows that the particle velocity $\mathbf{u}_{p}=d \mathbf{x} / d t$ can be written as a sum of the fluid velocity $\mathbf{u}_{f}$ and a "magnetic velocity" $\mathbf{u}_{m} \equiv \mathbf{F}_{m} / 3 \pi \mu D$. For a flow in the $x$ direction which varies parabolically in the $z$ direction, as shown in Fig. 1, the equations for the $x$ and $z$ components of the particle velocity become $\quad d x / d t=F_{m x} / 3 \pi \mu D+u_{0}\left(1-z^{2} / h^{2}\right)$ and $d z / d t$ $=F_{m z} / 3 \pi \mu D$. Dividing the first of these equations by the second, one obtains the inverse of the tangent to the particle trajectory

$$
\frac{d x}{d z}=\frac{F_{m x}}{F_{m z}}+\frac{3 \pi \mu D u_{0}}{F_{m z}}\left(1-\frac{z^{2}}{h^{2}}\right) .
$$

We introduce $F_{m 0}$ denoting the magnitude of the magnetization force at the channel centerline directly below the magnetic source (the origin in the coordinates of Fig. 1). Next we nondimensionalize Eq. (5) by defining $\overline{\mathbf{x}} \equiv \mathbf{x} / h$ and $\overline{\mathbf{F}}_{m} \equiv \overline{\mathbf{F}}_{m} / F_{m 0}$ :

$$
\frac{d \bar{x}}{d \bar{z}}=\frac{\bar{F}_{m x}}{\bar{F}_{m z}}+\frac{1}{\operatorname{Mn}_{p} \bar{F}_{m z}}\left(1-\bar{z}^{2}\right) .
$$

The dimensionless "particle magnetization number" $\mathrm{Mn}_{p}$ $\equiv F_{m 0} / 3 \pi \mu u_{0} D=u_{m 0} / u_{0}$ gives the ratio between the magnetization force and the drag force for a motionless particle at the origin or the ratio between its magnetic velocity $u_{m 0}$ and the fluid velocity $u_{0}$ at the origin. When $\bar{F}_{m 0 x}=0, \mathrm{Mn}_{p}$ alternatively denotes the tangent $d z / d x$ to a particle trajectory through the origin. The first-order ordinary differential equation (6) will, for various forms of the magnetization force $\overline{\mathbf{F}}_{m}$, be the subject of investigation in this paper.

\section{ANALYTICAL SOLUTIONS TO THE PARTICLE EQUATIONS OF MOTION}

The particle equation of motion [Eq. (6)] derived in the previous section will be solved for various forms of the magnetization force $\overline{\mathbf{F}}_{m}$. First a solution is derived for the case in which the force in the streamwise direction can be neglected and the force in the transversal direction only depends on the streamwise coordinate, i.e., $\overline{\mathbf{F}}_{m}=\bar{F}_{m}(\bar{x}) \hat{\mathbf{z}}$. In this case Eq. (6) becomes a separable differential equation, which can be solved by integration. As a special instance of this solution method, the particle motion under the influence of a constant magnetization force is obtained. The magnetization force exerted by a localized magnetic source many channel heights away generally satisfies the form $\overline{\mathbf{F}}_{m}=\bar{F}_{m}(\bar{x}) \hat{\mathbf{z}}$. This observation is used to calculate the particle trajectories in the field of a magnetic dipole. Finally an exact solution is obtained for a radial force that decays as a power of the distance to the source.

\section{A. Longitudinally varying transversal force}

When there is no magnetization force in the streamwise $x$ direction and the force in the transversal $z$ direction is inde-

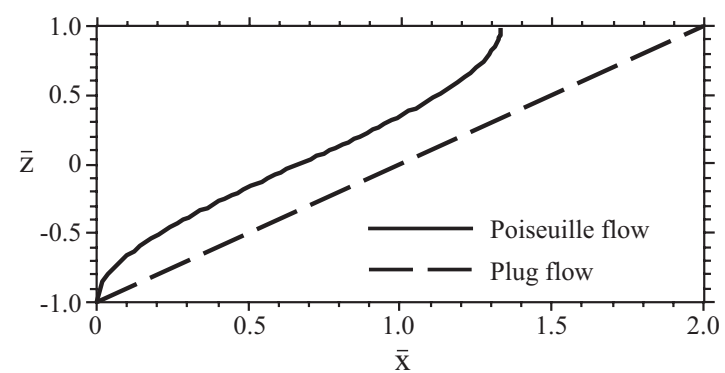

FIG. 2. The trajectories of particles under the influence of a constant force in the $z$ direction $\left(\mathrm{Mn}_{p}=1\right)$ in a parabolic Poiseuille flow and in a constant plug flow with the same centerline velocity.

pendent of $z$, i.e., $\overline{\mathbf{F}}_{m}=\bar{F}_{m}(\bar{x}) \hat{\mathbf{z}}$, Eq. (6) becomes

$$
\operatorname{Mn}_{p} \bar{F}_{m}(\bar{x}) \frac{d \bar{x}}{d \bar{z}}=1-\bar{z}^{2},
$$

which can be directly integrated to yield for a particle moving from $\left(\bar{x}_{0}, \bar{z}_{0}\right)$ to $(\bar{x}, \bar{z})$,

$$
\mathcal{M}\left(\bar{x}_{0}, \bar{x}\right)=\left(\bar{z}-\frac{\bar{z}^{3}}{3}\right)-\left(\bar{z}_{0}-\frac{\bar{z}_{0}^{3}}{3}\right) .
$$

Here we introduced the magnetization force to drag force ratio, integrated over the $\bar{x}$ coordinate of the particle trajectory:

$$
\mathcal{M}\left(\bar{x}_{0}, \bar{x}\right) \equiv \operatorname{Mn}_{p} \int_{\bar{x}_{0}}^{\bar{x}} \bar{F}_{m z}\left(\bar{x}^{\prime}\right) d \bar{x}^{\prime}=\frac{1}{3 \pi \mu D u_{0}} \frac{1}{h} \int_{x_{0}}^{x} F_{m z}\left(x^{\prime}\right) d x^{\prime} .
$$

Note from Eq. (8) that the vertical particle displacement $\bar{z}-\bar{z}_{0}$ depends solely on $\mathcal{M}$. This quantity thereby provides an intuitively sensible measure for the effectiveness of magnetic particle capture.

The solutions to the third-order polynomial equation Eq. (8) are given by

$$
\bar{z}=\left\{\begin{array}{c}
\zeta_{+}+\zeta_{-} \text {and } \\
-\frac{1}{2}\left[\left(\zeta_{+}+\zeta_{-}\right) \pm i \sqrt{3}\left(\zeta_{+}-\zeta_{-}\right)\right],
\end{array}\right.
$$

where $\zeta_{ \pm}=\left(v \pm \sqrt{v^{2}-1}\right)^{1 / 3}$ with $v=(3 / 2)\left(\bar{z}_{0}^{3} / 3-\bar{z}_{0}-\mathcal{M}\right)$ with the real-valued physical solution satisfying $-1 \leq \bar{z} \leq 1$.

For a constant force in the $z$ direction $\bar{F}_{m z} \equiv F_{m z} / F_{m 0}=1$ such that from Eq. (9) it follows that $\mathcal{M}=\operatorname{Mn}_{p}\left(\bar{x}-\bar{x}_{0}\right)$. From Eq. (8) then, a particle inserted at the lower channel wall $\bar{z}_{0}=-1$ will be the last to reach the upper wall at $\bar{z}=1$ after a distance $\bar{x}-\bar{x}_{0}=4 /\left(3 \mathrm{Mn}_{p}\right)$. The length $4 h /\left(3 \mathrm{Mn}_{p}\right)$ $=4 \pi \mu u_{0} D h / F_{m 0}$ therefore gives the distance after which all particles are filtered out upon application of a constant force. The particle trajectory described by Eq. (10) for $\mathcal{M}$ $=\operatorname{Mn}_{p}\left(\bar{x}-\bar{x}_{0}\right)$ is displayed in Fig. 2 for $\mathrm{Mn}_{p}=1$.

Inserting $n_{\text {in }}$ particles homogeneously distributed along the $z$ axis, we investigate for a given $\mathcal{M}\left(\bar{x}_{0}, \bar{x}\right)$ what fraction of particles will be captured by the magnetic field, i.e., what fraction will have reached $\bar{z}=1$ before $\bar{x}$. This capture efficiency (or collection efficiency) will be denoted by $\eta_{l}$, with 


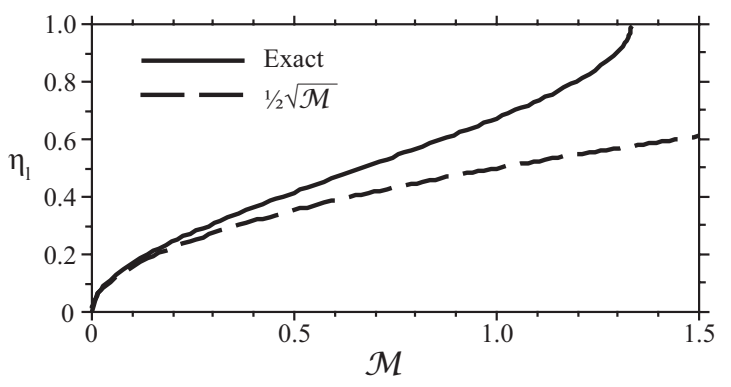

FIG. 3. The line capture efficiency $\eta_{l}$ as a function of $\mathcal{M}=\operatorname{Mn}_{p} \int \bar{F}_{m} d \bar{x}$ for particles under the influence of a force $\overline{\mathbf{F}}=\bar{F}_{m}(\bar{x}) \hat{\mathbf{z}}$.

the subscript $l$ referring to the injection of particles along a line. All particles inserted above a critical value $\bar{z}_{0}$ will reach $\bar{z}=1$, while all $n_{\text {out }}$ particles below $\bar{z}_{0}$ escape. The "line capture efficiency" $\eta_{l}$ is thus given by

$$
\eta_{l} \equiv \frac{n_{\text {in }}-n_{\text {out }}}{n_{\text {in }}}=\frac{\left(1-\bar{z}_{0}\right)}{2} .
$$

Here $\bar{z}_{0}$ is given by the solution to Eq. (8) with $\bar{z}=1$, which is the solution of Eq. (10) but now with $v$ $=\left.(3 / 2)\left(\bar{z}^{3} / 3-\bar{z}+\mathcal{M}\right)\right|_{\bar{z}=1}=3 \mathcal{M} / 2-1$. Expanding this solution in powers of $\sqrt{\mathcal{M}}$, one finds to lowest order

$$
\eta_{l}=\frac{1}{2} \sqrt{\mathcal{M}}, \quad \mathcal{M} \ll 1 .
$$

The exact capture efficiency and this approximation are both shown in Fig. 3 as a function of $\mathcal{M}$. Equation (12) shows that for small $\eta_{l}$ one has to increase the average magnetization force, or the length over which this force is applied, by a factor of 4 to double the capture efficiency. This is due to the parabolic nature of the flow. The flow velocity increases with distance from the wall, which adds to the difficulty of capturing particles from further away.

Because Eq. (12) holds only for small capture efficiencies it corresponds to particles close to the upper wall. Linearizing the parabolic flow near the wall yields $u_{f}=2 u_{0}(1-\bar{z})$. Equation (7) is then replaced by $\operatorname{Mn}_{p} \bar{F}_{m}(\bar{x}) d \bar{x} / d \bar{z}=2(1-\bar{z})$. This can be integrated for particles moving from $\left(\bar{x}_{0}, \bar{z}_{0}\right)$ to $(\bar{x}, 1)$ at the wall to yield $\mathcal{M}=1-2\left(\bar{z}_{0}-\frac{1}{2} \bar{z}_{0}^{2}\right)=\left(1-\bar{z}_{0}\right)^{2}$. The capture efficiency $\eta_{l}=\left(1-\bar{z}_{0}\right) / 2$ is thus given by Eq. (12) or for a constant force $\eta_{l}=\frac{1}{2} \sqrt{\operatorname{Mn}_{p}\left(\bar{x}-\bar{x}_{0}\right)}$. This rederivation of the result of Eq. (12) for a linearized flow velocity clearly shows that the square-root dependence can be attributed to the increasing flow velocity with the distance from the wall. In a small enough region close to the channel wall any particle magnetization force is approximately independent of $\bar{z}$ and most flows increase linearly with the distance from the wall, making Eq. (12) a quite general result. For a constant velocity "plug flow" Eq. (8) would be replaced by $\mathcal{M}=\bar{z}$. $-\bar{z}_{0}$ such that $\eta_{l}=\mathcal{M} / 2$, i.e., linearly in $\mathcal{M}$.

We note that the proportionality $\eta \propto \sqrt{\mathrm{Mn}_{p}}$ can be found in various experimental results. For example in Ref. [9] the magnetic field of two magnetized cylindrical wires is used, placed diametrically opposite each other and coaxial with the flow. For particles well below saturation, a roughly linear relationship between the capture efficiency and both $H$ and $1 / \sqrt{u_{0}}$ is found, supporting the result of Eq. (12). In Ref. [10] a magnetized coil is placed coaxially with the flow. A relation for the capture efficiency is found which can quite well be fitted with $\eta \propto 1 / \sqrt{u_{0}}$, again in agreement with the result of Eq. (12).

For a localized magnetic source with $\bar{d} \gg 1$, the magnetization force within the channel is approximately constant in the $z$ direction, i.e., $\bar{F}_{m z}=\bar{F}_{m z}(\bar{x})$. In order to be able to use the above results however, the term $\bar{F}_{m x} / \bar{F}_{m z}$ in Eq. (6) should be negligible in comparison with $\left(1-\bar{z}^{2}\right) / \mathrm{Mn}_{p} \bar{F}_{m z}$. Strictly speaking near $\bar{z}=1$ this only holds for $\bar{F}_{m x}=0$. However, under the influence of any nonzero $\bar{F}_{m z}$, particles this close to the wall will be swiftly captured. If one is thus not interested in the exact particle motion close to the wall, on an integral scale the term $\bar{F}_{m x} / \bar{F}_{m z}$ can be neglected when everywhere in the domain $\bar{F}_{m x} \ll 1 / \mathrm{Mn}_{p}=3 \pi \mu u_{0} D / F_{m 0}$ or $F_{m x} \ll F_{D}$. In this approximation the particle motion is the combined uncoupled motion of that of the fluid and that of the velocity in the $z$ direction induced by the magnetic field. This approach is also taken in [4], where it is used to derive an approximate solution for the particle motion in the field of a cylinder homogeneously magnetized perpendicular to its axis. In the following section we will use it to calculate the particle motion in the field of a magnetic dipole.

\section{B. Magnetic dipole}

We proceed to solve the motion of undersaturated magnetic particles in the field of a magnetic dipole for $\bar{d} \gg 1$. As discussed, in this case the first term on the right-hand side of Eq. (6) can be neglected and the solution of the previous section can be used. The magnetic field at a position vector $\mathbf{r}$ emanating from the dipole is given by

$$
\mathbf{H}(\mathbf{r})=\frac{m}{4 \pi r^{3}}[3(\hat{\mathbf{m}} \cdot \hat{\mathbf{r}}) \hat{\mathbf{r}}-\hat{\mathbf{m}}] .
$$

Note that again a hat is used to denote a unit vector, e.g., $\hat{\mathbf{m}}=\mathbf{m} / m$ with $m=|\mathbf{m}|$. With $H^{2}=\left(m / 4 \pi r^{3}\right)^{2}\left[3(\hat{\mathbf{m}} \cdot \hat{\mathbf{r}})^{2}+1\right]$, the magnetization force density $\mu_{0} \chi \nabla H^{2} / 2$ below saturation becomes

$$
\mathbf{f}_{m}(\mathbf{r})=\frac{\mu_{0} \chi}{2}\left(\frac{m}{4 \pi}\right)^{2} \frac{6}{r^{7}}\left\{(\hat{\mathbf{m}} \cdot \hat{\mathbf{r}}) \hat{\mathbf{m}}-\left[1+4(\hat{\mathbf{m}} \cdot \hat{\mathbf{r}})^{2}\right] \hat{\mathbf{r}}\right\} .
$$

Both the field $\mathbf{H}$ of Eq. (13) and this force density are displayed in Fig. 4(b). Note that for a dipole oriented in the $y$ direction $\hat{\mathbf{m}} \cdot \hat{\mathbf{r}}=0$ for $y=0$ such that $\mathbf{f}_{m}=$ $-3 \mu_{0} \chi(m / 4 \pi)^{2}\left(\hat{\mathbf{r}} / r^{7}\right)$ for $y=0$. For this radial magnetization force an exact analytical solution will be derived in the next section. Allowing for an arbitrary orientation of the dipole, we introduce the spherical coordinates $\theta$ and $\phi$ representing the azimuthal angle between the dipole moment and the $z$ axis and the polar angle with the $x$ axis, respectively, such that $\hat{\mathbf{m}}=\sin \theta \cos \phi \hat{\mathbf{x}}+\sin \theta \sin \phi \hat{\mathbf{y}}+\cos \theta \hat{\mathbf{z}}$. Evaluating Eq. 


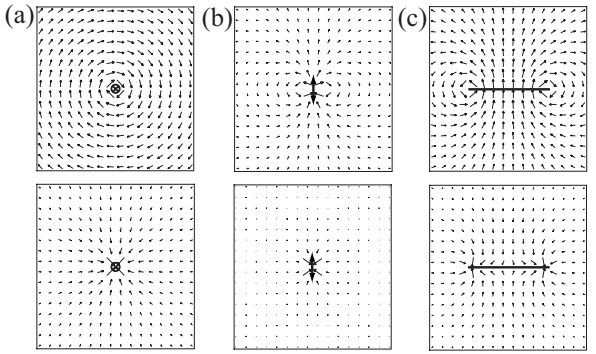

FIG. 4. The magnetic field $\mathbf{H}$ (top) and associated magnetization force density below saturation $\mu_{0} \chi \nabla H^{2} / 2$ (bottom) of (a) a current carrying wire as an example of a power-law force, (b) a magnetic dipole, and (c) a circular current loop (side view). Note that the length of the vectors is scaled proportional to their magnitude to the power $1 / 5$.

(14) for a dipole located at $d \hat{\mathbf{z}}$ we obtain with $\mathbf{r}=-d \hat{\mathbf{z}}$ for the force at the origin (in the coordinates of Fig. 1) $F_{m 0}$ $=\left(\mu_{0} \chi / 2\right) V(m / 4 \pi)^{2}\left(18 \cos \theta^{2}+6\right) / d^{7}$. Performing the integration of Eq. (9) for $y=0$ we obtain

$$
\begin{aligned}
\mathcal{M}= & C \bar{x}\left(5 \cos ^{2} \theta+2+\sin ^{2} \theta \cos ^{2} \phi\right) \\
& \times\left(\frac{15 \arctan \bar{x} / \bar{d}}{16 \bar{x} \bar{d}^{6}}+\frac{15}{16 \bar{d}^{5} \bar{r}^{2}}+\frac{5}{8 \bar{d}^{3} \bar{r}^{4}}+\frac{1}{2 \bar{d} \bar{r}^{6}}\right) \\
& +\frac{C}{\bar{r}^{8}}\left[\cos \theta \sin \theta \cos \phi\left(6 \bar{d}^{2}-\bar{r}^{2}\right)\right. \\
& \left.+3 \bar{d} \bar{x}\left(\cos ^{2} \theta-\sin ^{2} \theta \cos ^{2} \phi\right)\right],
\end{aligned}
$$

where $\bar{r}^{2}=\bar{x}^{2}+\bar{d}^{2}$ and $C \equiv\left(\mu_{0} \chi / 2\right)\left(1 / 3 \pi \mu u_{0} D\right)(m / 4 \pi)^{2} / h^{7}$ $=\operatorname{Mn}_{p}(\theta) \bar{d}^{7} /\left(18 \cos ^{2} \theta+6\right)$. Note that $M n_{p}$ now is a function of the angle $\theta$ between the dipole moment and the $z$ axis. Equation (15) evaluated between $\bar{x}_{0}$ and $\bar{x}$, in combination with the solution of Eq. (10), gives an analytical expression $\bar{z}(\bar{x})$ for the particle trajectories in the magnetic field of an arbitrarily oriented magnetic dipole (for $\bar{d} \gg 1$ and $\bar{F}_{m x}$ $\ll 1 / \mathrm{Mn}_{p}$ ).

We are now in a position to answer the question for which orientation of a magnetic dipole the most particles will be captured. Evaluating Eq. (15) between the limits $\bar{x}_{0}=-\infty$ and $\bar{x}=\infty$ we obtain for $\mathcal{M}_{\infty} \equiv \lim _{x^{\prime} \rightarrow \infty} \mathcal{M}\left(-x^{\prime}, x^{\prime}\right)$

$$
\mathcal{M}_{\infty}=\frac{15 \pi C}{16 \bar{d}^{6}}\left(5 \cos ^{2} \theta+2+\cos ^{2} \phi-\cos ^{2} \phi \cos ^{2} \theta\right),
$$

which is plotted in Fig. 5. From this we find that $\mathcal{M}_{\infty}$ is a maximum for $\theta=0$ or $\pi$, i.e., by aligning the magnetic dipole in the $\pm z$ direction. Using the result $\eta_{l}=\sqrt{\mathcal{M}} / 2$ of Eq. (12), which is valid for $\mathcal{M} \ll 1$, we find that the capture efficiency can be expressed as

$$
\eta_{l}=f(\theta, \phi) \sqrt{\operatorname{Mn}_{p}(\theta) \bar{d}}, \quad \mathcal{M} \ll 1 \ll \bar{d},
$$

with

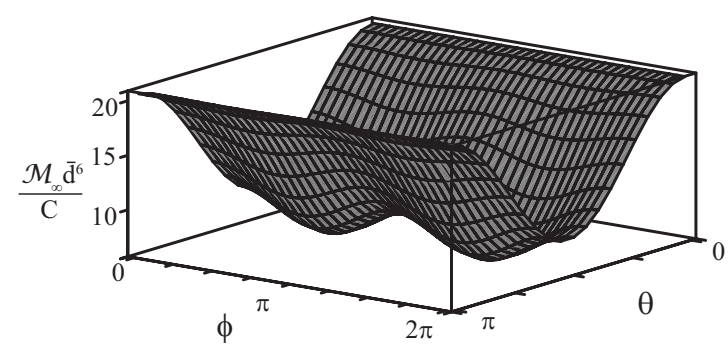

FIG. 5. $\mathcal{M}_{\infty} \bar{d}^{6} / C$ as a function of the dipole angles $\theta$ and $\phi$.

$$
\begin{aligned}
f(\theta, \phi) & \\
= & \sqrt{(5 \pi / 128)} \\
& \times \sqrt{\left(5 \cos ^{2} \theta+2+\cos ^{2} \phi-\cos ^{2} \phi \cos ^{2} \theta\right) /\left(3 \cos ^{2} \theta+1\right)} .
\end{aligned}
$$

Specifically for a dipole oriented in the $x, y$, and $z$ directions, respectively, we obtain

$$
\begin{aligned}
& \eta_{l}^{(x)}=\sqrt{\frac{15 \pi}{128}} \sqrt{\operatorname{Mn}_{p}^{(x)} \bar{d}}, \quad \mathcal{M} \ll 1 \ll \bar{d}, \\
& \eta_{l}^{(y)}=\sqrt{\frac{10 \pi}{128}} \sqrt{\operatorname{Mn}_{p}^{(y)} \bar{d}}, \quad \mathcal{M} \ll 1 \ll \bar{d}, \\
& \eta_{l}^{(z)}=\sqrt{\frac{35 \pi}{512}} \sqrt{\operatorname{Mn}_{p}^{(z)} \bar{d}}, \quad \mathcal{M} \ll 1 \ll \bar{d},
\end{aligned}
$$

where the superscripts $(x),(y)$, and $(z)$ refer to the corresponding orientation of the dipole. In Fig. 6 these relations are compared to the capture efficiency obtained from Eq. (10), which is exact in the limit $\bar{d} \rightarrow \infty$. From Eq. (14) it follows that at a fixed distance $r$ from the dipole, the magnetization force parallel to the dipole moment is four times as high as that perpendicular to the dipole moment. This implies that for the same magnetic dipole moment $\mathrm{Mn}_{p}^{(z)}$ $=4 \mathrm{Mn}_{p}^{(y)}=4 \mathrm{Mn}_{p}^{(x)}$. We thus find that the capture efficiencies for the same magnetic dipole moment are related via $\eta_{l}^{(z)}$ $=\sqrt{7 / 3} \eta_{l}^{(x)}=\sqrt{7} \eta_{l}^{(y)}$ for $\mathcal{M} \ll 1 \ll \bar{d}$.

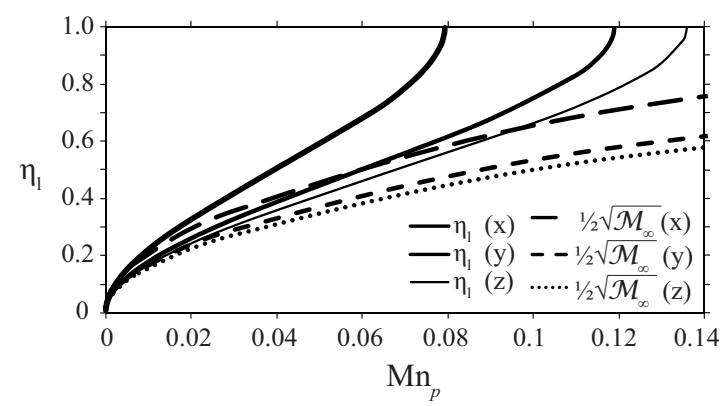

FIG. 6. The capture efficiency $\eta_{l}$ for the field of a magnetic dipole oriented in the $x, y$, and $z$ directions for the specific case $\bar{d}=80 / 7$. The analytical solution derived for $\bar{d} \gg 1$ is compared to the approximation $\sqrt{\mathcal{M}_{\infty}} / 2$ valid for $\mathcal{M}_{\infty} \ll 1 \ll \bar{d}$. 


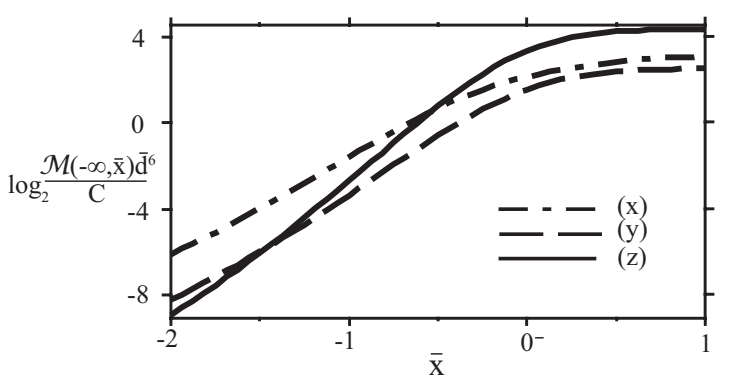

FIG. 7. $\log _{2}\left[\mathcal{M}(-\infty, \bar{x}) \bar{d}^{6} / C\right]$ for a magnetic dipole orientation in the $x, y$, and $z$ directions.

Typically the multipole expansion of the magnetic field associated with a current density distribution $\mathbf{J}(\mathbf{r})$ confined to a region of dimension $L$ contains a dominant dipole term. At a distance $d \gg L$ the magnetic field then resembles that of a magnetic dipole. For such a localized current distribution the magnetic dipole moment $\mathbf{m} \equiv(1 / 2) \int \mathbf{J}(\mathbf{r}) \times \mathbf{r} d^{3} r$ can thus for $d \gg L$ and $d \gg h$ best be directed toward the flow in order to maximize the capture efficiency. An example is a circular wire of radius $a$ carrying a current $I$, which has a dipole moment $\mathbf{m}=\pi a^{2} I \hat{\mathbf{n}}$, with $\hat{\mathbf{n}}$ the normal to the plane of the current. For $d \gg 2 a$ and $d \gg h$ it follows that the capture efficiency is maximized by orienting $\hat{\mathbf{n}}$ toward the flow.

To obtain more information on the particle behavior in the field of a magnetic dipole, we look at the quantity $\mathcal{M}($ $-\infty, \bar{x}$ ). From Eq. (8) the transversal displacement $\bar{z}-\bar{z}_{0}$ of particles at $\bar{x}$ that entered from $\bar{x}_{0}=-\infty$ depends solely on this quantity. For a dipole aligned with the $x, y$, or $z$ axis, $\mathcal{M}($ $-\infty, \bar{x})$ is displayed in Fig. 7. From this figure we observe that in total most particles will be captured for a dipole in the $z$ direction, i.e., $\lim _{\bar{x} \rightarrow \infty}[\mathcal{M}(-\infty, \bar{x})]=\mathcal{M}_{\infty}$ is the largest for this orientation. Far downstream the magnet however $\mathcal{M}($ $-\infty, \bar{x})$ is smallest for this orientation such the least particles will be captured. This complies with the anisotropic magnetization force distribution shown in Fig. 4. Aside from yielding the highest capture efficiency, alignment toward the flow thus also minimizes the spatial extent over which particles are captured. In filtering applications this means a smaller channel length can be used, in magnetic drug targeting it implies better focusing of the medicine.

\section{Power-law forces}

With a magnetic field whose magnitude varies as $H$ $=A^{\prime} / r^{n^{\prime}}$, where $r$ is the distance to a line or point source, according to Eq. (2) a magnetization force density $\mathbf{f}_{m}=$ $-A / r^{n} \hat{\mathbf{r}}$ is associated. Here $A=\mu_{0} \chi n^{\prime} A^{\prime 2}$ and $n=2 n^{\prime}+1$ for undersaturated and $A=\mu_{0}(\chi /|\chi|) M_{s a t} n^{\prime} A^{\prime}$ and $n=n^{\prime}+1$ for saturated particles. The dimensionless magnetization force is in this case given by

$$
\overline{\mathbf{F}}_{m}=-\left(\frac{\bar{d}}{\bar{r}}\right)^{n} \hat{\mathbf{r}} .
$$

For saturated and undersaturated particles, respectively, this represents the following:

(i) For $n=2$ and $n=3$ : the force of an infinitely long wire carrying a current $I$ such that $A^{\prime}=I / 2 \pi$.

(ii) For $n=3$ and $n=5$ : the force of an infinitely long cylinder of radius $R_{c}$ with a uniform magnetization $\mathbf{M}_{c}$ perpendicular to its axis [4] such that $A^{\prime}=M_{c} R_{c}^{2} / 2$. Or a line dipole $[5,14]$, two antiparallel wires carrying a current $I$ an infinitesimal distance $b$ apart [13], with dipole strength $P$ $=I b / 2 \pi$ such that $A^{\prime}=P$. It also represents a magnetic monopole with pole strength $p$ such that $A^{\prime}=p / 4 \pi \mu_{0}$.

(iii) For $n=7$ it represents for $\bar{y}=0$ the force on undersaturated particles of a magnetic dipole $\mathbf{m}$ oriented in the $y$ direction such that $A^{\prime}=m / 4 \pi$.

The magnetic field and force $\overline{\mathbf{F}}$ of a current carrying wire are shown in Fig. 4(a). We first analyze the magnetic particle motion in the presence of a line source at a distance $d$ from and parallel to the centerline of the flow. In the coordinates of Fig. $1 \bar{F}_{m x}=0$ and $\bar{F}_{m z}=-(\bar{z}-\bar{d}) \bar{d}^{n} /|\bar{z}-\bar{d}|^{n+1}=\bar{d}^{n} /(\bar{d}-\bar{z})^{n}$ such that the particle equation of motion [Eq. (6)] is readily integrated to yield

$$
\bar{x}-\bar{x}_{0}=\left.\frac{1}{\operatorname{Mn}_{p}} \frac{(\bar{d}-\bar{z})^{n+1}}{\bar{d}^{n}} \frac{-n^{2}+\bar{z}^{2} n^{2}+2 \bar{z} \bar{d} n-5 n+3 n \bar{z}^{2}-6+2 \bar{z} \bar{d}+2 \bar{z}^{2}+2 \bar{d}^{2}}{(n+3)(n+2)(n+1)}\right|_{\bar{z}_{0}} ^{\bar{z}} .
$$

In the limit $\bar{d} \rightarrow \infty$ the distance $\left.\left(\bar{x}-\bar{x}_{0}\right)\right|_{\bar{z}_{0}=-1} ^{\bar{z}=1}$ after which all particles are captured becomes $4 /\left(3 \mathrm{Mn}_{p}\right)$. As expected, this is the same length as in the case of a constant force. Note that because for saturated particles the magnetization force is linear in the applied field, several similar terms for different values of $\bar{d}, \mathrm{Mn}_{p}$, and/or $n$ can be added to the right-hand side of Eq. (22). In this way the obtained result can, e.g., describe the trajectories of saturated particles in the plane of symmetry of the configuration studied in Ref. [24]. Orienting a line source perpendicular to the flow

$$
\overline{\mathbf{F}}_{m}=-\bar{d}^{n} \frac{\bar{x} \hat{\mathbf{x}}+(\bar{z}-\bar{d}) \hat{\mathbf{z}}}{\left[\bar{x}^{2}+(\bar{z}-\bar{d})^{2}\right]^{(n+1) / 2}},
$$

such that the particle equation of motion [Eq. (6)] becomes

$$
\frac{d \bar{x}}{d \bar{z}}=\frac{\bar{x}}{\bar{z}-\bar{d}}-\frac{\left[\bar{x}^{2}+(\bar{z}-\bar{d})^{2}\right]^{(n+1) / 2}}{\operatorname{Mn}_{p} \bar{d}^{n}(\bar{z}-\bar{d})}\left(1-\bar{z}^{2}\right) .
$$

To solve Eq. (24) we introduce the angle $\gamma$ shown in Fig. 1. This is the same approach as that followed in Ref. [5] for 


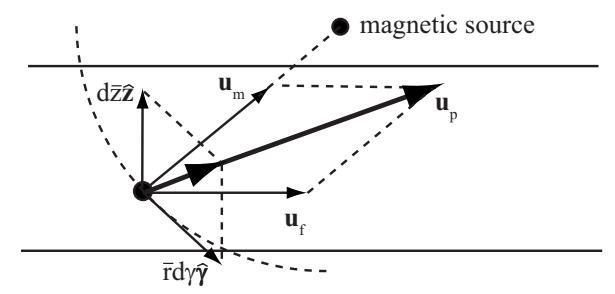

FIG. 8. A schematic diagram showing the direction in which a magnetic particle moves in a radial magnetization force.

the particular case of $n=5$. Changing coordinates from $\bar{x}$ to $\gamma=\arctan \frac{-\bar{x}}{\bar{z}-\bar{d}}$, Eq. (24) becomes

$$
\frac{(\bar{z}-\bar{d})^{2}}{\left(\cos ^{2} \gamma\right)} \frac{d \gamma}{d \bar{z}}=\frac{1-\bar{z}^{2}}{\operatorname{Mn}_{p} \bar{d}^{n}}\left[(\bar{z}-\bar{d})^{2}\left(\tan ^{2} \gamma\right)+(\bar{z}-\bar{d})^{2}\right]^{(n+1) / 2}
$$

Using $1+\tan ^{2} \gamma=\cos ^{-2} \gamma$ this can be written as

$$
\frac{d \gamma}{d \bar{z}}=\frac{1}{\operatorname{Mn}_{p} \bar{d}^{n}} \frac{(\bar{z}-\bar{d})^{n-1}\left(1-\bar{z}^{2}\right)}{(\cos \gamma)^{n-1}} .
$$

In terms of the radial coordinate $\bar{r}=(\bar{d}-\bar{z}) / \cos \gamma$ this equation can be written as

$$
\frac{1}{\bar{r}} \frac{d \bar{z}}{d \gamma}=\frac{\operatorname{Mn}_{p} \bar{F}_{m}}{1-\bar{z}^{2}} .
$$

We note that this relation could also have been obtained in a different way, from Fig. 8. From Eq. (4) we know that the particle velocity $\mathbf{u}_{p}$ is given by the sum of the fluid velocity $\mathbf{u}_{f}$ and the magnetic velocity $\mathbf{u}_{m}=\mathbf{F}_{m} / 3 \pi \mu D$. This is in the same direction as the vector spanned by the resulting particle displacements $d \bar{z} \hat{\mathbf{z}}$ in the $z$ direction and $\bar{r} d \gamma \hat{\gamma}$ tangential to the force such that $d \bar{z} / r d \gamma=u_{m} / u_{f}=F_{m} / 3 \pi \mu D u_{f}$ and Eq. (27) follows.

Equation (26) can be integrated to yield

$$
\begin{aligned}
\operatorname{Mn}_{p} \bar{d} \int_{\gamma_{i}}^{\gamma}\left(\cos \gamma^{\prime}\right)^{n-1} d \gamma^{\prime}= & -\frac{1}{\bar{d}^{n-1}} \frac{(\bar{z}-\bar{d})^{n}}{(n+2)(n+1) n}\left(-n^{2}+n^{2} \bar{z}^{2}\right. \\
& \left.+2 \bar{z} \bar{d} n-3 n+n \bar{z}^{2}+2 \bar{d}^{2}-2\right)\left.\right|_{\bar{z}_{i}} ^{\bar{z}_{f}}
\end{aligned}
$$

Performing the integration on the left-hand side and solving for $\bar{z}$ yields an exact solution to Eq. (6) for the particle trajectories $\bar{z}(\gamma)$. To obtain $\eta_{l}$ from Eq. (28), one inserts the limits $\gamma_{i}=-\pi / 2$ and $\gamma=\pi / 2$ and seeks for a solution to the resulting algebraic equation satisfying $-1 \leq \bar{z} \leq 1$. The integral of Eq. (28) can then be written as

$$
\int_{-\pi / 2}^{\pi / 2}\left(\cos \gamma^{\prime}\right)^{n-1} d \gamma^{\prime}=\sqrt{\pi} \frac{\Gamma\left(\frac{n}{2}\right)}{\Gamma\left(\frac{n}{2}+\frac{1}{2}\right)}=B\left(\frac{n}{2}, \frac{1}{2}\right) .
$$

The beta function [25] $B(n / 2,1 / 2)$ is plotted in Fig. 9. The values of $B(n / 2,1 / 2)$ for $n=2,3,4,5,6$, and 7 are

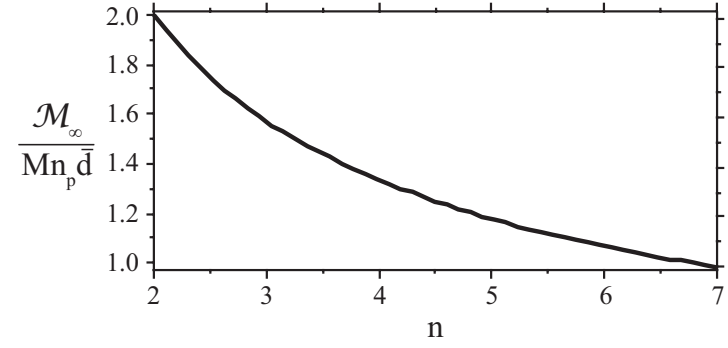

FIG. 9. $\mathcal{M}_{\infty} / \operatorname{Mn}_{p} \bar{d}=B(n / 2,1 / 2)$ for a power-law force $\overline{\mathbf{F}}_{m}=-(\bar{d} / \bar{r})^{n} \hat{\mathbf{r}}$.

given by $2, \pi / 2,4 / 3,3 \pi / 8,16 / 15$, and $5 \pi / 16$, respectively. Note that, using Eq. (27), the solution method deployed here can be used for any radial force that can be written as a product of a function of $r$ and a function of $\gamma$.

Although the exact analytical result of Eq. (28) contains all the information on the particle trajectories, we take the approach of the previous section and investigate the limit $\bar{d}$ $\gg 1$. In this limit, the force in the $z$ direction becomes $\bar{F}_{m z}$ $=\bar{d}^{n+1} /\left(\bar{x}^{2}+\bar{d}^{2}\right)^{(n+1) / 2}$. Changing coordinates we find for $\mathcal{M}_{\infty}=\operatorname{Mn}_{p} \int_{-\infty}^{\infty} \bar{F}_{m z}(\bar{x}) d \bar{x}$

$$
\mathcal{M}_{\infty}=\operatorname{Mn}_{p} \bar{d} \int_{-\pi / 2}^{\pi / 2}\left(\cos \gamma^{\prime}\right)^{n-1} d \gamma^{\prime}=B\left(\frac{n}{2}, \frac{1}{2}\right) \operatorname{Mn}_{p} \bar{d} .
$$

This quantity is displayed in Fig. 9. The approximation $\eta_{l}=\sqrt{\mathcal{M}_{\infty}} / 2$ of Eq. (12) yields

$$
\eta_{l}=\frac{1}{2} \sqrt{B\left(\frac{n}{2}, \frac{1}{2}\right) \mathrm{Mn}_{p} \bar{d}}, \quad \mathcal{M} \ll 1 \ll \bar{d} .
$$

In Fig. 10 a comparison is made between the exact capture efficiency $\eta_{l}$ from Eq. (28) and the approximation $\eta_{l}$ $=\sqrt{\mathcal{M}_{\infty}} / 2$ for the specific case of $n=5$ and $\bar{d}=80 / 7$. Note that because in the limit $\bar{d} \gg 1$ the force becomes independent of $\bar{z}$, the capture efficiency in this case becomes symmetric around $\eta_{l}=1 / 2$. Due to the increasing magnetization force with increasing $\bar{z}$, however, the exact result for $\bar{d}=80 / 7$,

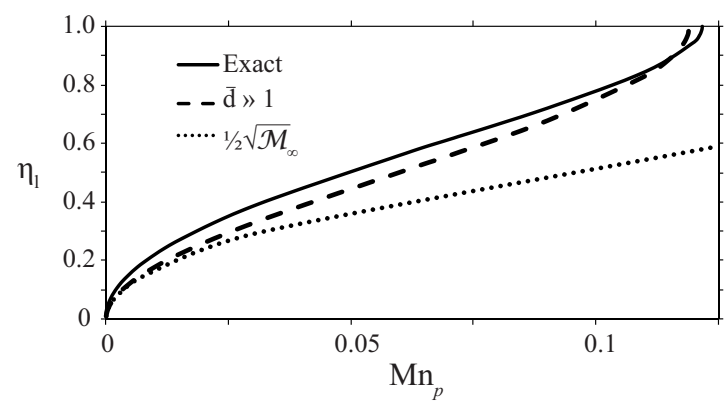

FIG. 10. The capture efficiency $\eta_{l}$ for a force $\overline{\mathbf{F}}_{m}=-(\bar{d} / \bar{r})^{n} \hat{\mathbf{r}}$, with $n=5$ and $\bar{d}=80 / 7$ as calculated from the exact solution to the particle equations of motion, the result valid for $\bar{d} \gg 1$, and the ap-

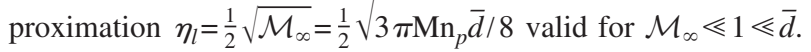


shown in Fig. 10, still shows some asymmetry.

\section{CONVERSION TO CYLINDRICAL GEOMETRY}

So far a two-dimensional flow in the $x$ direction has been considered with a velocity magnitude that varies parabolically in the $z$ direction. This includes the steady laminar flow between two parallel plates $u(\bar{z})=3\langle u\rangle\left(1-\bar{z}^{2}\right) / 2$ but also the flow in a specific $\bar{y}=\bar{y}_{1}$ plane of a cylindrical channel for which $u(\bar{y}, \bar{z})=2\langle u\rangle\left(1-\bar{y}^{2}-\bar{z}^{2}\right)$. By rewriting this for $\bar{y}=\bar{y}_{1}$ as $u\left(\bar{y}_{1}, \bar{z}\right)=2 \alpha^{2}\langle u\rangle\left[1-(\bar{z} / \alpha)^{2}\right]$, with $\alpha=\sqrt{1-\bar{y}_{1}^{2}}$, the flow velocity is manifestly parabolic in the $z$ direction for $-\alpha \leq \bar{z} \leq \alpha$ with a peak flow velocity $2\langle u\rangle \alpha^{2}$. When particle motion in the $y$ direction can be neglected, particles traverse a parabolic flow profile in the transverse direction. In this case expressions obtained for the capture efficiency $\eta_{l}\left(\mathrm{Mn}_{p}, \bar{d}\right)$ in two dimensions can be used to determine the capture efficiency $\eta_{c y l} \equiv \eta_{c y l}\left(\mathrm{Mn}_{p}, \bar{d}\right)$ for particles inserted homogeneously distributed in a cylindrical domain. This can be done, e.g., for a line source oriented in the $y$ direction, for which there is no particle magnetization force in the $y$ direction. For a localized source at $\bar{y}=0$ generally a force in the $y$ direction exists for nonzero $\bar{y}$. This force however becomes negligible compared to the force in the $z$ direction when the source is placed far away from the flow, i.e., for $\bar{d} \gg 1$. In the remainder of this section it will be assumed that the particle motion in the $y$ direction can be neglected. Integrating the line capture efficiency $\eta_{l}$ over a cross section and dividing by the corresponding area

$$
\eta_{c y l}=\frac{1}{\pi} \int_{-1}^{1} \eta_{l}\left(\frac{\mathrm{Mn}_{p}}{1-\bar{y}^{2}}, \frac{\bar{d}}{\sqrt{1-\bar{y}^{2}}}\right) 2 \sqrt{1-\bar{y}^{2}} d \bar{y},
$$

where $\mathrm{Mn}_{p}$ and $\bar{d}$ have been rescaled with the lower peak velocity and channel height for nonzero $\bar{y}$. Next we evaluate Eq. (32) for $\eta_{l} \equiv \eta_{l}\left(\mathrm{Mn}_{p}, \bar{d}\right)=c \mathrm{Mn}_{p}^{\alpha} \bar{d}^{\beta}$, which was, for example, shown to be a valid parametrization with $\alpha=\beta=1 / 2$ for $\quad \mathcal{M} \ll 1 \ll \bar{d}$. With $\quad \eta_{l}\left[\mathrm{Mn}_{p} /\left(1-\bar{y}^{2}\right), \bar{d} / \sqrt{1-\bar{y}^{2}}\right]=\eta_{l} /(1$ $\left.-\bar{y}^{2}\right)^{\alpha+\beta / 2}$ and realizing that the capture efficiency exceeds 1 for $\bar{y}^{2} \geq 1-\eta_{l}^{1 /(\alpha+\beta / 2)} \equiv \bar{y}_{c}^{2}$

$$
\begin{aligned}
\eta_{c y l}= & \frac{4}{\pi} \int_{0}^{1} \min \left(\frac{\eta_{l}}{\left(1-\bar{y}^{2}\right)^{\alpha+\beta / 2}}, 1\right) \sqrt{1-\bar{y}^{2}} d \bar{y} \\
= & \frac{4 \eta_{l}}{\pi} \int_{0}^{\bar{y}_{c}}\left(1-\bar{y}^{2}\right)^{(1-2 \alpha-\beta) / 2} d \bar{y}+\frac{4}{\pi} \int_{\bar{y}_{c}}^{1} \sqrt{1-\bar{y}^{2}} d \bar{y} \\
= & \frac{4}{\pi}\left[\int_{0}^{\bar{y}_{c}} \eta_{l} \times\left(1-\bar{y}^{2}\right)^{(1-2 \alpha-\beta) / 2} d \bar{y}-\frac{1}{2} \bar{y}_{c} \sqrt{1-\bar{y}_{c}^{2}}\right. \\
& \left.-\frac{1}{2} \arcsin \bar{y}_{c}\right]+1 .
\end{aligned}
$$

When $\bar{y}_{c} \approx 1$ the integral of Eq. (33) can be evaluated ignoring the fact that $\eta_{l}$ is restricted to 1 , yielding

$$
\eta_{c y l} \approx \eta_{l} \frac{2 \Gamma\left(\frac{3}{2}-\alpha-\beta / 2\right)}{\sqrt{\pi} \Gamma(2-\alpha-\beta / 2)}=\frac{2 \eta_{l}}{\pi} B\left(\frac{1}{2}, \alpha+\beta / 2\right) .
$$

This provides a valid approximation only when the integrand in Eq. (33) does not diverge too fast, i.e., when $2 \alpha$ $+\beta$ is not too large. For $\alpha=\beta=1 / 2$ this approximation gives a reasonably small error provided $1-\bar{y}_{c} \ll 1$.

\section{COMPUTATIONAL RESULTS}

Computational simulations in a cylindrical geometry have been performed to investigate the accuracy of the correction factors $\eta_{c y l} / \eta_{l}$ derived in the previous section and to extend the obtained analytical results beyond their region of applicability. The analysis of the optimal orientation of a magnetic dipole for magnetic particle capture is with simulations extended to $\bar{d}=O(1)$. Finally the capture efficiency for the magnetic field of a circular current loop is investigated.

The simulations have been performed, using commercial fluid dynamics software FLUENT 6.0 (Fluent Inc., Lebanon, $\mathrm{NH})$. The fully developed laminar flow of a Newtonian fluid has been simulated in a cylindrical domain, such that a Poiseuille flow resulted. A one-way coupled discrete particle model was used with a drag force [26] that becomes equal to the Stokes drag force for $\operatorname{Re}_{p} \leq 0.1$.

A channel diameter $2 h=7 \mathrm{~mm}$, an average flow velocity $\langle u\rangle=u_{0} / 2=0.1 \mathrm{~m} / \mathrm{s}, \quad$ a kinematic viscosity $\nu=3.5$ $\times 10^{-6} \mathrm{~m}^{2} / \mathrm{s}$, and density $\rho_{f}=1000 \mathrm{~kg} / \mathrm{m}^{3}$ were used, such that $\operatorname{Re}=200$ and roughly corresponding to a strongly idealized blood flow in a large artery.

A magnetic source was placed halfway the length of the channel. By varying its distance to the centerline, $\bar{d}$ was varied. Particles of various sizes were inserted such that $\mathrm{Mn}_{p}$ was varied. Per particle size $n_{i n}=266$ particles were inserted homogeneously distributed over the circular inlet. The particles were assumed to be undersaturated everywhere in the domain.

The source strength in all simulations was chosen such that for a particle with $D=0.5 \mu \mathrm{m}$ and $\chi=3$, a value of $\mathrm{Mn}_{p}=1 / 2$ resulted. The particle diameters were successively halved to yield diameters ranging from $D=1 / 2 \mu \mathrm{m}$ to $(1 / 2)^{8} \mu \mathrm{m} \approx 3.9 \mathrm{~nm}$ corresponding to values of $\mathrm{Mn}_{p}$ ranging from $\mathrm{Mn}_{p}=1 / 2$ to $(1 / 2)^{15}$. By counting the number of particles $n_{\text {out }}$ leaving the domain through the outlet, the capture efficiency $\eta \equiv\left(n_{\text {in }}-n_{\text {out }}\right) / n_{\text {out }}$ as a function of both $\mathrm{Mn}_{p}$ and $\bar{d}$ was obtained for several magnetic field configurations.

The domain was chosen large enough to ensure a negligible magnetization force at both the inlet and the outlet. This was confirmed by observing no change in capture efficiency when the domain size was increased.

\section{A. Comparison with analytical results}

First we consider simulations with $d=4 \mathrm{~cm}$ such that $\bar{d}$ $\equiv d / h \approx 11.4$ and the particle displacement in the $y$ direction is small compared to the displacement in the $z$ direction. In this case the correction factors $\eta_{c y l} / \eta_{l}$ of Sec. IV can be used to approximately convert the analytical results obtained in 

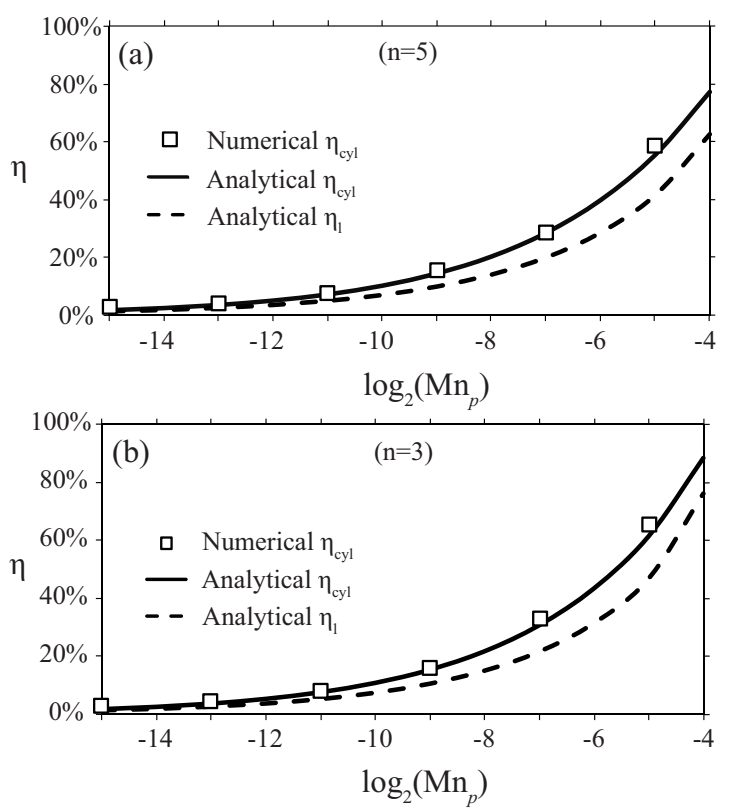

FIG. 11. The capture efficiency $\eta$ for (a) a current carrying wire (power-law force with $n=3$ ) and (b) a magnetized cylinder (powerlaw force with $n=5$ ) obtained computationally with $\bar{d}=80 / 7$ is compared with $\eta_{l}$ from the two-dimensional exact analysis and $\eta_{c y l}$ corrected for the cylindrical geometry.

two dimensions to the three-dimensional cylindrical domain. First we look at a power-law source for which an exact analytical solution to the particle equation of motion has been obtained in Sec. III C. Figure 11 compares $\eta_{l}$ obtained from Eq. (28) with data from the simulations for $n=3$ and $n=5$. It can be seen that $\eta_{l}$ severely underestimates the capture efficiency. This was expected from the fact that $\eta_{l}$ gives the fraction of particles captured with $\bar{y}=0$, where both the fluid velocity and the distance to the upper wall is largest of all values of $\bar{y}$. In Fig. 11 also $\eta_{c y l}$ is displayed, which is obtained using the correction of Eq. (34) with $\alpha=\beta=1 / 2$. After this correction, good correspondence is obtained with the computational results. The remaining differences can be attributed to the fact that the parametrization $\eta_{l}=c \operatorname{Mn}_{p}^{\alpha} \bar{d}^{\beta}$, with $\alpha=\beta=1 / 2$ used in the correction $\eta_{c y l} / \eta_{l}$, is only valid for $\bar{d} \gg 1$ and $\mathcal{M} \ll 1$.

The correction factor $\eta_{c y l} / \eta_{l}$ that is used in Fig. 11 is plotted in Fig. 12 along with the approximation of Eq. (35) $2 B(1 / 2,3 / 4) / \pi=4 \sqrt{2} \Gamma(3 / 4)^{2} / \pi^{3 / 2} \approx 1.53$ for $1-\bar{y}_{c} \ll 1$. The two correction factors approach each other in the limit $\mathrm{Mn}_{p}$ $\rightarrow 0$, for which $\bar{y}_{c} \rightarrow 1$.

A similar comparison between the analytical result and simulations has been performed for various orientations of a magnetic dipole. The results are shown in Fig. 13. The analytical result used for the capture efficiency was obtained using the expression for $\mathcal{M}_{\infty}$ of Eq. (16) valid for $\bar{d} \gg 1$.

\section{B. Varying the source distance}

Simulations with the source distance varying from $d$ $=4 \mathrm{~mm}, 5 \mathrm{~mm}, 1 \mathrm{~cm}, 2 \mathrm{~cm}$ to $4 \mathrm{~cm}$, with $h=3.5 \mathrm{~mm}$ yielded $\bar{d}=d / h=8 / 7,10 / 7,20 / 7,40 / 7$, and 80/7. From the results of

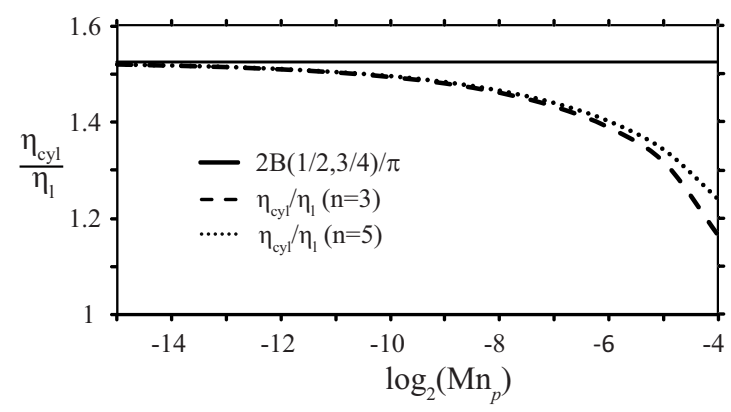

FIG. 12. The correction factor $\eta_{c y l} / \eta_{l}$ used for a current carrying wire $(n=3$ and $\bar{d}=80 / 7)$ and a magnetized cylinder $(n=5$ and $\bar{d}=80 / 7)$ compared to the approximation $2 B(1 / 2,3 / 4) / \pi$ for $1-\bar{y}_{c} \ll 1$.

these simulations, performed for several different magnetic fields, the capture efficiency was found to be parametrized quite effectively by a power of $\mathrm{Mn}_{p}$, i.e.,

$$
\eta=g \mathrm{Mn}_{p}^{q}
$$

with $g$ and $q$ functions of $\bar{d}$. Using only data points corresponding to $0.04 \leq \eta \leq 0.96$, at least four data points remained per simulation which had a (Pearson productmoment) correlation $r>0.997$ with the fit of Eq. (36). It should be noted that by our choice of successively halving the particle diameters, linearly the data points are unevenly distributed between $0 \leq \eta \leq 1$ with a higher density for smaller values of $\eta$.

In Fig. 14 the result of the fit of Eq. (36) for the prefactor $g(\bar{d})$ and exponent $q(\bar{d})$ are displayed for various power-law forces: a current carrying wire (line source with $n=3$ ), a cylinder magnetized perpendicular to its axis (line source with $n=5$ ), and a magnetic monopole (point source with $n$ $=5)$. The first thing to note is that the exponent $q(\bar{d})$ tends to a value of $1 / 2$ for $\bar{d} \gg 1$ in accordance with the theory developed in Sec. III A. More interestingly, for smaller values of $\bar{d}$ the exponent $q$ is lower than this asymptotic value. In this case more than a magnetization force quadrupling is needed to double the capture efficiency. This is due to the fact that for a more than linearly decaying force strength, a doubling

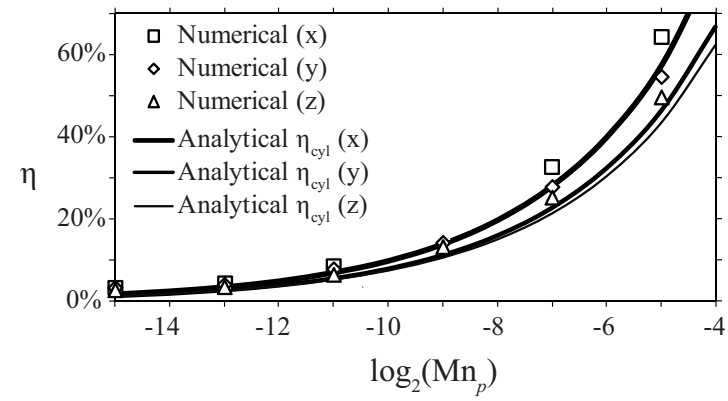

FIG. 13. The capture efficiency $\eta$ for a magnetic dipole in the $x$, $y$, and $z$ directions, obtained computationally for $\bar{d}=80 / 7$, is compared to the capture efficiency obtained from the analytical result $\eta_{l}$ (for $\bar{d} \gg 1$ ) and $\eta_{c y l}$ which is corrected for the cylindrical geometry. 

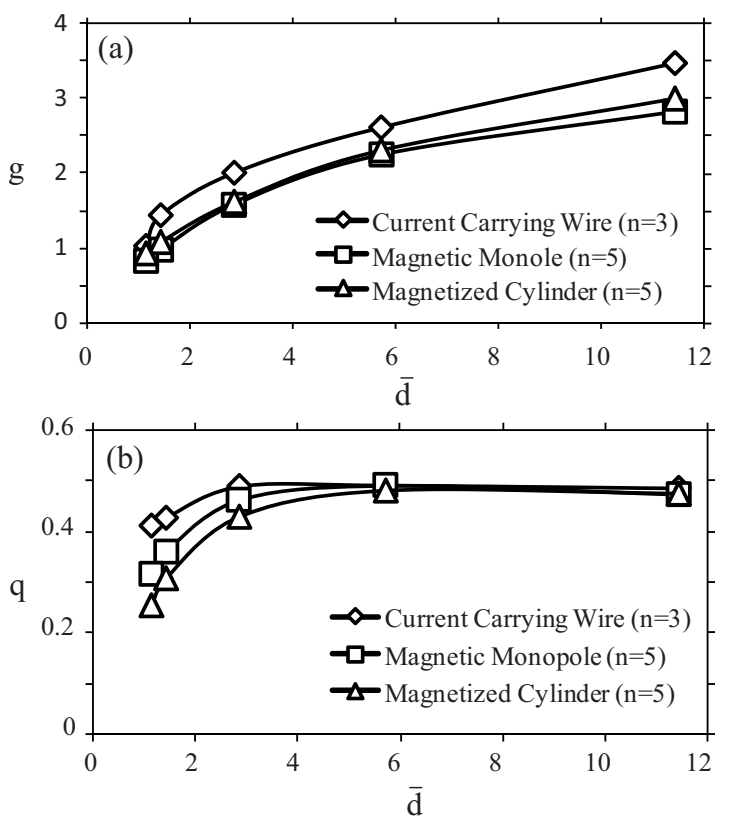

FIG. 14. The computational result of the parametrization $\eta=g(\bar{d}) \mathrm{Mn}_{p}^{q(\bar{d})}$ for various power-law sources.

of the force does not double the distance over which the force is larger than a certain value. The prefactor $g(\bar{d})$ was found to be an increasing function of $\bar{d}$, which can be explained by the increasing extent over which the magnetization force acts with increasing $\bar{d}$.

The curves for a magnetic monopole and a magnetized cylinder, a point source with $n=5$ and a line source with $n$ $=5$, respectively, closely resemble each other. For any specific value of $\mathrm{Mn}_{p}$ however the capture efficiency is slightly higher for the latter magnetic source. This can be explained by the fact that the distance particles travel toward the wall is for a point source everywhere equal to or larger than for a line source, for which there is no particle motion in the $y$ direction.

In Ref. [5] a fit $\eta \propto \mathrm{Mn}_{p}^{0.45}$ is made of the exact analytical result [Eq. (28)] for a power-law force with $n=5$ and $\bar{d}=3$. The value $q=0.45$ is close to that obtained in our simulations, as can be seen from Fig. 14. In Ref. [13] an advectiondiffusion equation is solved for the particle concentration using the same magnetic force. For $\bar{d}=1.1$ the obtained capture efficiency can be fitted by $\eta \propto \mathrm{Mn}_{p}^{0.27}$, corresponding quite well with our result in Fig. 14.

For a magnetic dipole, the results of the parametrization of Eq. (36) are displayed in Fig. 15. Again we note that for a dipole of the same magnetic moment, the value of $\mathrm{Mn}_{p}$ for the $z$ orientation is four times higher than that of the $x$ and $y$ orientations of the dipole. Taking this into account we see that more particles are captured for a $z$ orientation than for an $x$ orientation. For an $x$ orientation in turn, the capture efficiency is higher than for a $y$ orientation. The computational results therefore show that the conclusion obtained in Sec. III B for $\bar{d} \gg 1$ continues to hold for values of $\bar{d}=O(1)$.

A dipole orientation toward the flow was also used in the experiments reported in Ref. [7], involving a rectangular
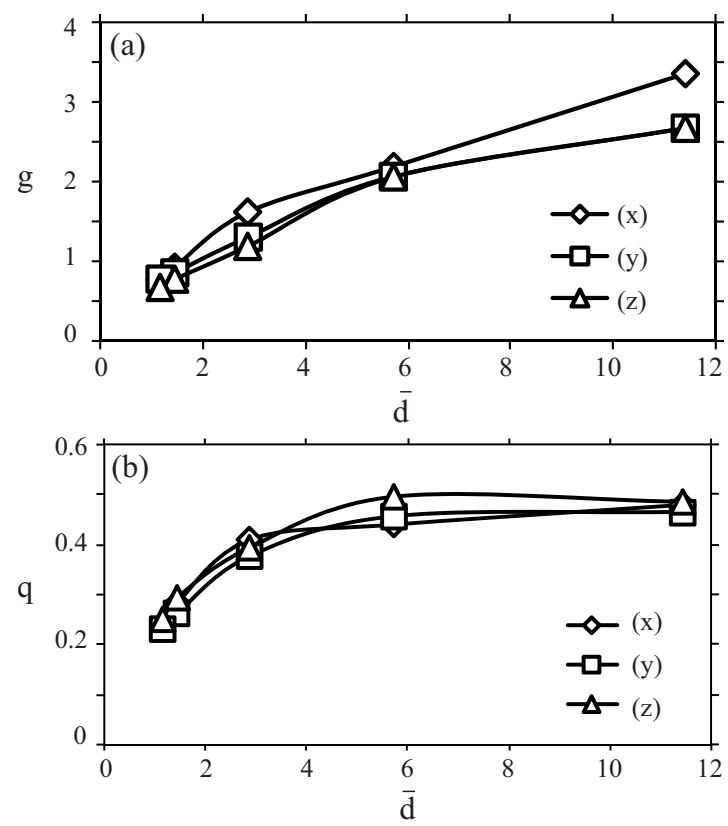

FIG. 15. The computational result of the parametrization $\eta=g(\bar{d}) \mathrm{Mn}_{p}^{q(\bar{d})}$ for magnetic dipole orientations in the $x, y$, and $z$ directions.

flow domain. Particles moving from the channel wall opposite the wall closest to the dipole traverse an approximately parabolic flow profile such that, for high capture efficiencies, it should be possible to make a comparison with the present work. Above $\eta \approx 20 \%$ an approximate dependence $\eta$ $\propto \mathrm{Mn}_{p}^{0.48}$ is reported for $\bar{d}=1.75$ [7]. Figure 15 shows for a $z$ dipole a somewhat smaller value of $q \approx 0.32$. It should however be noted that reported deviation from a pure magnetic dipole field and the rectangular geometry make an accurate comparison difficult.

Finally, the particle motion in the magnetic field of a circular current loop was investigated computationally. An analytical expression for the magnetic field [27] was used to calculate the associated magnetization force. Both the field and the force on an undersaturated particle are shown in Fig. 4(c). A loop radius of $a=1 \mathrm{~cm}$ was used, yielding $\bar{a} \equiv a / \mathrm{h}$ $=20 / 7$. This extra geometrical parameter and the direction of the loop normal oriented in the $z$ direction were not varied. Interesting to note from the results of the parametrization of Eq. (36), displayed in Fig. 16, is the initial decrease in $g(\bar{d})$ with $\bar{d}$. This is due to the spatial distribution of the magnetization force of a circular current loop. By moving the loop away from the channel while keeping $\mathrm{Mn}_{p}$ constant, the spatial extent over which the force acts on the particles increases. The fringe fields very close to the loop, shown in Fig. 4, however die out much faster than the field along the symmetry axis through the loop. Initially this latter effect decreases the capture efficiency. For larger values of $\bar{d}$ the former effect increases the efficiency again. Because the spatial extent of these fringe fields is of the order of the loop radius $a$, this would predict for fixed $\mathrm{Mn}_{p}$ a minimum capture efficiency for $\bar{d}=O(\bar{a})$, which can indeed be observed from Fig. 16. Decreasing $\bar{d}$ from $\bar{a}$ to $0.4 \bar{a}$ in the simulations, 

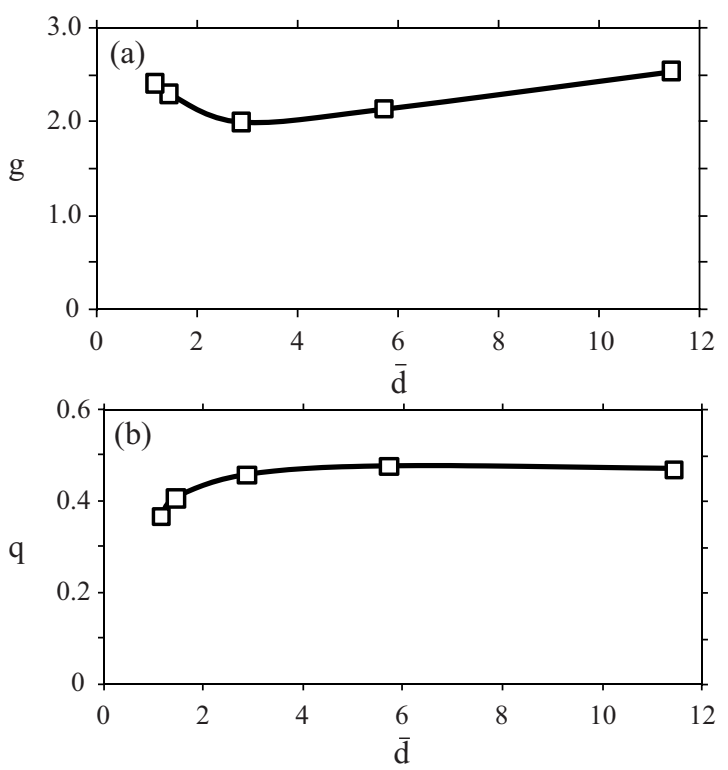

FIG. 16. The computational result of the parametrization $\eta=g(\bar{d}) \mathrm{Mn}_{p}^{q(\bar{d})}$ for a circular current loop with radius $\bar{a}=a / h$ $=20 / 7$.

the value of $\mathrm{Mn}_{p}$ needed to capture the same fraction of particles decreased by significantly a factor of approximately 3. A similar effect might be relevant when using electromagnets or superconducting magnets for particle capture.

\section{CONCLUDING REMARKS}

Under the assumption of small particle Reynolds numbers and negligible gravitational and lift forces, the velocity of a spherical magnetic particle in a flow with velocity $\mathbf{u}_{f}$ under the influence of a magnetization force $\mathbf{F}_{m}$ can be written $\mathbf{u}_{p}$ $=\mathbf{u}_{f}+\mathbf{F}_{m} / 3 \pi \mu D=\mathbf{u}_{f}+u_{0} \operatorname{Mn}_{p} \overline{\mathbf{F}}_{m}$. For a flow in the $x$ direction which varies parabolically in the $z$ direction, this particle equation of motion was solved for a force in the transversal $z$ direction only depending on the streamwise $\bar{x}$ coordinate, i.e., $\overline{\mathbf{F}}_{m}=\bar{F}_{m}(\bar{x}) \hat{\mathbf{z}}$. In terms of the quantity $\mathcal{M}=\operatorname{Mn}_{p} \int \bar{F}_{m} d \bar{x}$ the particle capture efficiency was found to be approximated by

$$
\eta=\frac{1}{2} \sqrt{\mathcal{M}}, \quad \mathcal{M} \ll 1 .
$$

This result shows that a four times higher magnetization force or a four times smaller drag force is required to double the capture efficiency, in agreement with various reported experimental and computational results $[5,7,9,10]$. The square-root behavior of Eq. (37) was found to be a consequence of the fact that the flow velocity initially increases linearly with the distance from the wall. Because this holds for almost any flow, the result applies very generally. For a relative magnetic source distance $\bar{d} \gg 1$ the magnetization force in the channel can often be approximated by $\overline{\mathbf{F}}_{m}$ $=\bar{F}_{m z}(\bar{x}) \hat{\mathbf{z}}$, making the obtained result valuable for various magnetic fields. For $\bar{d} \gg 1$ the most relevant contribution to the magnetic field is generally a dipole term, which was investigated analytically. It was found that an orientation of the dipole moment toward the flow yields the highest capture efficiency. An exact solution for all $\bar{d}$ was obtained for the particle motion in a radial magnetic field, applicable to, e.g., the field of a current carrying wire and a uniformly magnetized cylinder.

Under the assumption of negligible particle displacement in the $y$ direction a correction factor was constructed to convert the capture efficiency from a planar to a cylindrical geometry. Using this correction good correspondence was obtained for $\bar{d} \gg 1$ between the analytical results and computational simulations in a cylindrical geometry. From these simulations, the capture efficiency was found to be effectively parametrized by $\eta=g \mathrm{Mn}_{p}^{q}$, where $g$ and $q$ are the functions of the relative magnetic source distance $\bar{d}$. For $\bar{d}$ $\gg 1$ the exponent $q$ was found to be approximately $1 / 2$. Because mostly data points for low capture efficiencies have been used in these fits, this confirms the result of Eq. (37). For all investigated magnetic field configurations, $q$ was found to be below $1 / 2$ for smaller values of $\bar{d}$ due to the more than linear decay with distance of the associated magnetization forces. The prefactor $g$ was found to be an increasing function of $\bar{d}$. This can be attributed to the increasing spatial extent over which the magnetization force acts with increasing $\bar{d}$. For a circular current loop, however, $g$ was found to have a minimum at $d$ roughly corresponding to the loop radius due to rapidly decaying fringe fields close to the loop.
[1] J. Oberteuffer, IEEE Trans. Magn. 10, 223 (1974).

[2] A. S. Lübbe et al., Cancer Res. 56, 4686 (1996).

[3] C. Alexiou et al., Cancer Res. 60, 6641 (2000).

[4] E. P. Furlani and K. C. Ng, Phys. Rev. E 73, 061919 (2006).

[5] K. Nandy, S. Chaudhuri, R. Ganguly, and I. K. Puri, J. Magn. Magn. Mater. 320, 1398 (2008).

[6] V. Badescu, V. Murariu, O. Rotariu, and N. Rezlescu, Magn. Electr. Sep. 8, 23 (1996).

[7] A. Sinha, R. Ganguly, A. K. De, and I. K. Puri, Phys. Fluids 19, 117102 (2007).

[8] S. Nishijima et al., IEEE Trans. Appl. Supercond. 18, 874
(2008).

[9] H. Chen et al., Phys. Med. Biol. 52, 6053 (2007).

[10] M. O. Avilés et al., J. Magn. Magn. Mater. 311, 306 (2007).

[11] M. O. Avilés et al., J. Magn. Magn. Mater. 293, 605 (2005).

[12] Gh. Iacob, O. Rotariu, N. J. C. Strachan, and U. O. Häfeli, Biorheology 41, 599 (2004).

[13] C. Mikkelsen and H. Bruus, Lab Chip 5, 1293 (2005).

[14] N. Modak, A. Datta, and R. Ganguly, Microfluid. Nanofluid. 6, 647 (2009).

[15] N. Pamme, Lab Chip 7, 1644 (2007).

[16] E. P. Furlani, J. Appl. Phys. 99, 024912 (2006). 
[17] E. P. Furlani, J. Phys. D 40, 1313 (2007).

[18] H. Lee, A. M. Purdon, V. Chu, and R. M. Westervelt, Nano Lett. 4, 995 (2004).

[19] H. Lee, A. M. Purdon, and R. M. Westervelt, Appl. Phys. Lett. 85, 1063 (2004).

[20] E. Mirowski, J. Moreland, A. Zhang, and S. E. Russek, Appl. Phys. Lett. 86, 243901 (2005).

[21] Handbook of Chemistry and Physics, 67th ed., edited by R. C. Weast, M. J. Astle, and W. H. Beyer (CRC Press, Boca Raton, FL, 1986).

[22] H. Brenner and J. Happel, J. Fluid Mech. 4, 195 (1958).
[23] P. G. Saffman, J. Fluid Mech. 22, 385 (1965).

[24] H. Chen, M. D. Kaminski, and A. J. Rosengart, Med. Eng. Phys. 30, 1 (2008).

[25] Handbook of Mathematical Functions with Formulas, Graphs, and Mathematical Tables, edited by, M. Abramowitz and I. A. Stegun (Dover, New York, 1972).

[26] S. A. Morsi and A. J. Alexander, J. Fluid Mech. 55, 193 (1972)

[27] D. J. Jackson, Classical Electrodynamics, 3rd ed. (Wiley, New York, 1998). 